\title{
Performance Analysis Cooperative Networks of AF over Nakagami-m Fading Channels with SC and MRC
}

\author{
Ali Abdulwahhab \\ Mohammed \\ Department of Electronic and \\ Information Engineering \\ Huazhong University of \\ Science and Technology \\ Wuhan 430074, China
}

\author{
$\mathrm{Li} \mathrm{Yu}$ \\ Department of Electronic and \\ Information Engineering \\ Huazhong University of \\ Science and Technology \\ Wuhan 430074, China
}

\author{
Manar Al-Kali \\ Department of Electronic and \\ Information Engineering \\ Huazhong University of \\ Science and Technology \\ Wuhan 430074, China
}

\begin{abstract}
In this paper the performance of amplify and forward relay system with Maximum Ratio Combining (MRC) and Selective Combining (SC) over Nakagami-m fading channels is studied by considering both MRC and SC schemes at the destination, we derive the cumulative density function (CDF), probability density function (PDF) and moment generating function (MGF) for the multiple relay AF network with single half duplex In addition, we derive the exact Symbol Error Rate (SER) of M-ary phase-shift keying (M-PSK), in Nakagami -m fading environment. Simulation results are presented to show that system performance of the differential $\mathrm{SC}$ is comparable to the MRC at the receivers, and improves with the increase of repeaters, and different decoding methods have different effects on system performance.
\end{abstract}

\section{Keywords}

Cooperative Networks, AF, Maximal ratio combining, Selective Combining, Nakagami -m fading channels

\section{INTRODUCTION}

Cooperative wireless networks are a promising technology for future communications systems, with the development of wireless network technology. Wireless cooperative communication network had been recently proposed as a technique that can significantly increase the system capacity and diversity gain in wireless networks without requiring much more resources [1-4].

Nakagami-m fading distribution covers a wide range of fading scenarios via the $m(m>0.5)$ parameter, which includes the Rayleigh $m=1$ as a special case [5]. In [6-7] the authors adopted the relay selection method in AF relaying systems and operate in half duplex mode. The derivation of new closed-form expressions for the cumulative distribution function (CDF) and moment generating function (MGF) of end-to-end SNR in non-regenerative opportunistic relaying over Nakagami-m fading provide useful statistical analysis on the optimality of wireless networks. In this paper we submitted the analysis to far more via deriving a novel closedform expression for ASEP when MRC and SC techniques are employed at the destination. Several works has been discussed on for example, the authors of [8] presented the analysis of AF cooperative network in Nakagami-m fading environment with $\mathrm{MRC}$ and $\mathrm{N}$ single antenna relays. However in this work the analysis and the derive of the exact sample error rate (SER) performance of maximal ratio combining receivers over Nakagami-m fading channels based on PDF, CDF and MGF of the out dual channel MRC, and comparison between M-PSK and M-QAM modulation schemes are provided
In [9] the authors considered the relay node operate in $\mathrm{AF}$ cooperative network half duplex mode. The SER for M-ary phase shift keying (M-PSK) and M-ary quadrature amplitude modulation (M-QAM) schemes is discussed. The approximated closed-form expression of ergodic capacity is derived for fixed multiple-relay network under independent not necessarily identically distributed Nakagami-m and Rician fading. The MRC receiver is used at the destination to realize the desired diversity.

In [10-11] the error performance of the MRC with transmits antenna selection and receiver maximal-ratio combining (TAS/MRC) scheme are investigated in independent Nakagami-m fading channels for arbitrary $\mathrm{m}$. and attained from two different approaches. Then the exact symbol error rate (SER) expressions are attained as a multiple infinite sum based on the moment generating function (MGF) method for $\mathrm{M}$-ary phase-shift keying (M-PSK) and M-ary quadrature amplitude modulation (M-QAM) [12]. The asymptotic SER expressions reveal a diversity order equal to the product of the $\mathrm{m}$ parameter. The authors in [13] derived a conditional lower bound for the error rate and the outage probability $\left(\mathrm{P}_{\text {out }}\right)$ of the cooperative diversity networks over independent and nonidentical Nakagami-m fading channels with $\mathrm{AF}$ relaying and maximum ratio combining (MRC) at the destination node.

This paper presents the analysis of AF cooperative network in Nakagami-m fading environment with Special cases include maximum ratio combining (MRC), and selection combining (SC), which all achieve the same sum diversity even when the combined branches are correlated, or, have SNRs following different PDFs. We also provide analysis and derivation of the exact sample error rate (SER) performance of MRC and $\mathrm{SC}$ receivers over Nakagami-m fading channels based on the derived PDF, CDF and MGF of the out dual channel MRC. The novel obtained expression is then used to Study the effect of diversity reception error on the performance and we will be considering on the network that is studied by both MRC and SC schemes at the destination. with M-ary phase-shift keying (M-PSK) and M-ary with different values of the ratio of the decision after a quadrature phase over Nakagami -m Fading Channels.

The remainder of this paper is organized as follows. Section 2 describes the system model. We derive the PDF, CDF ,MGFand SER of the out dual channel considering MRC and $\mathrm{SC}$ at the destination,in Section 3 ,Section 4 numerical results in Section 5 conclusions. 


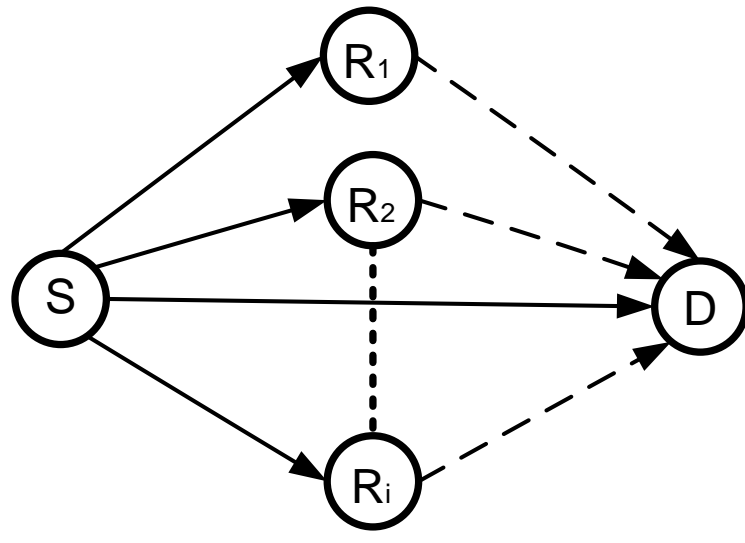

Fig 1: System model of Cooperative network with the $L$ relays, $\mathbf{R}_{1}, \mathbf{R}_{2}, \cdots, \mathbf{R}_{\mathrm{i}}$ is selected to with direct path.

\section{SYSTEM MODEL}

We describe in this section the cooperative network distributed the contract Source $S$ communicates with the destination node $D$ with the help of $i^{\text {th }}$ relay nodes, $\left(R_{1}, R_{2}, \ldots \ldots, R_{i}\right)$ using amplify - and - forward protocol as shown in Figure 1.

Each node is equipped with an antenna in the independent flat Nakagami-m fading channels and operates in half duplex mode. The signals are transmitting as an orthogonal presumably either in time or frequency. The corresponding fading coefficients for the $S \rightarrow D, S \rightarrow R_{i}$ and $R_{i} \rightarrow D$ are $h_{S D}, h_{S R_{i}}$ and $h_{R_{i} D}$, are fading channel respectively. Due to Nakagami -m fading Channels.

The signals received at the destination and the $i^{\text {th }}$ relay, the system model can be described by the following set of equation as follows:

$$
\begin{aligned}
& y_{S R_{i}}=h_{S R_{i}} \sqrt{P_{s}} x+\eta_{S R_{i}} \\
& y_{S D}=h_{S D} \sqrt{P_{s}} x+\eta_{S D}
\end{aligned}
$$

$x$ is the transmitted signal, $P_{S}$ is transmitted source power, $\eta_{S D}$ and $\eta_{S R_{i}}$ as well as $\eta_{R_{i} D}$ are all additive White Gaussian noises (AWGN) with variances $N o$.

In the second phase, the source $\mathrm{S}$ is suspended to transmit the signal, and the relay terminal $\mathrm{R}_{\mathrm{i}}$ directly carries on the power amplification to the source signal. After the forward signal, the destination $\mathrm{D}$ to receive the signal from the relay terminal $\mathrm{R}_{\mathrm{i}}$ can be expressed as:

$$
y_{R_{i} D}=h_{R_{i} D} \sqrt{P_{r}} x_{r}+\eta_{R_{i} D}
$$

where, $h_{R_{i} D}$ is the channel fading coefficient of $R_{i} \rightarrow D$ link, $\eta_{R_{i} D}$ is $0, P_{r}$ is the received power at the $\mathrm{i}^{\text {th }}$ relay, The AF mode is to amplify and transmit the signal received by the relay $R_{i}$ and $D$, set $G$ for its amplification factor, the signal can be transmitted to the relay $\mathrm{R}_{\mathrm{i}}$ :

$$
x_{r}=G y_{S R_{i}}
$$

The signal transmitted by the relay $\mathrm{R}_{\mathrm{i}} x_{r}=\bar{G} y_{S R_{i}}{ }^{\mathrm{t}}$ the $x_{r}$ is the information on the relay. In order to meet the power constraint, the received signal $y_{S R_{i}}$ power is normalized.

$$
\bar{G}=\sqrt{\frac{1}{\left|h_{S R_{i}}\right|^{2} P_{S}+N_{O}}}
$$

The destination receives the transmission as:

$$
y_{R_{i} D}=h_{R_{i} D} \mathrm{G} y_{S R_{i}}+\eta_{R_{i} D}
$$

where

$$
G=\sqrt{\frac{P_{r}}{\left|h_{S R_{i}}\right|^{2} P_{S}+N_{O}}}
$$

The $y_{S R_{i}}$ in the equation (6) can be obtained:

$$
y_{R_{i} D}=h_{R_{i} D} h_{S R_{i}} \mathrm{G} \sqrt{P_{s}} x+h_{R_{i} D} \mathrm{G} y_{S R_{i}}+\eta_{R_{i} D}
$$

Since, solving the exact expressions in (8), we can estimate the instantaneous end-to-end SNR can be expressed as follows:

$$
\gamma_{i}=\frac{\gamma_{S R_{i}} \gamma_{R_{i} D}}{\gamma_{S R_{i}}+\gamma_{R_{i} D}+1}
$$

Let us define the effective instantaneous SNRs for source to destination, source to the $\mathrm{i}^{\text {th }}$ relay and $\mathrm{i}^{\text {th }}$ relay to destination links as $\gamma_{S R_{i}}=\left|h_{S R_{i}}\right|^{2} P_{S} / N_{o}, \gamma_{R_{i} D}=\left|h_{R_{i} D}\right|^{2} P_{r} / N_{o}$ and $\gamma_{S D}=\left|h_{S D}\right|^{2} P_{S} / N_{o}$ respectively. For the direct link .

\section{PERFORMANCE ANALYSIS SER}

In this section, we present network performance through analyzing the Symbol Error Rate (SER) over Nakagami-m fading channels for any number of relays with MRC and SC receiver at the destination.

\subsection{Performance of MRC Combination System}

In this section, we study Cooperative Networks of $\mathrm{AF}$ over Nakagami-m fading Channels in relaying with MRC at the destination. The channel state information operates in halfduplex mode. Let direct link $S \rightarrow D$, and a relay link $S \rightarrow R_{i}$ and $R_{i} \rightarrow D$. MRC the end to end of the SNR combination can be expressed as.

$$
\gamma_{r m s}=\gamma_{S D}+\frac{\gamma_{S R_{i}} \gamma_{R_{i} D}}{1+\gamma_{S R_{i}}+\gamma_{R_{i} D}}
$$

Since, solving the exact expressions in (10) can be complicated; we derive an upper bound from (10) as:

$$
\gamma_{i}^{u p}=\min \left(\gamma_{S R_{i}}, \gamma_{R_{i} D}\right) \geq \frac{\gamma_{S R_{i}} \gamma_{R_{i} D}}{1+\gamma_{S R_{i}}+\gamma_{R_{i} D}}
$$

Equivalent SNR can be expressed as:

$$
\gamma_{D}^{u p} \leq \gamma_{S D}+\sum_{i=1}^{R} \gamma_{i}^{u p}
$$

Assuming that $\gamma_{S D}$ and $\gamma_{i}^{u p}$ are independent, the MGF of $\gamma_{D}^{u p}$ can be written as. 


$$
M_{\gamma_{D}^{u p}}(s)=M_{\gamma_{S D}}(s) \prod_{i=1}^{R} M_{\gamma_{i}}(s)
$$

Where $\mathrm{m}$ is the fading parameter, $\mathrm{As} \mathrm{m}_{\mathrm{SD}}, \mathrm{m}_{\mathrm{SRi}}$ and $\mathrm{m}_{\mathrm{RiD}}$ the Nakagami -m distribution. As well as the channel parameter $h_{S D}$ is subject to Nakagami-m distribution, which can be obtained:

$$
M_{\gamma_{S D}}(s)=\left(1+s \frac{\bar{\gamma}_{S D}}{m_{S D}}\right)^{-m_{S D}}
$$

For Nakagami-m fading channel, $\bar{\gamma}_{S D}=\varepsilon_{2} \times\left|h_{S D}\right|^{2} P_{S} / N_{o}$ Link for average $\mathrm{SNR}, \varepsilon_{2}$ is the energy consumed at the destination.

In order to find the MGF for the $\mathrm{S}-\mathrm{R}-\mathrm{D}$ path, it needs to derive the corresponding PDF of CDF. It is shown that when $\gamma_{S R_{i}}$ and $\gamma_{R_{i} D}$ are independent the CDF of $\gamma_{i}^{u p}=\min \left(\gamma_{S R_{i}}, \gamma_{R_{i} D}\right)$ can be expressed as [14].

$$
\begin{gathered}
F_{\gamma_{i}^{u p}}(\gamma)=1-\left[1-P_{r}\left(\gamma_{S R_{i}} \geq \gamma\right)\right]\left[1-P_{r}\left(\gamma_{R_{i} D} \geq \gamma\right)\right] \\
F_{\gamma_{i}^{u p}}(\gamma)=1-\left[1-F_{S R_{i}}(\gamma)\right]\left[1-F_{R_{i} D}(\gamma)\right] \\
F_{S R_{i}}(\gamma)=\Gamma\left(m, \frac{m}{\bar{\gamma}_{S R_{i}}^{2}} \gamma\right) \\
F_{R D}(\gamma)=\Gamma\left(m, \frac{m}{\bar{\gamma}_{R D}^{2}} \gamma\right)
\end{gathered}
$$

$F_{S R}(\gamma)$ And $F_{R D}(\gamma)$ are the CDF from the source to the $i^{\text {th }}$ relay and from the $i^{\text {th }}$ relay to the destination respectively.

Let:

$$
\begin{aligned}
& \bar{\gamma}_{S R_{i}}=c_{1} \bar{\gamma}_{C} \\
& \bar{\gamma}_{R_{i} D}=c_{2} \bar{\gamma}_{C}
\end{aligned}
$$

where $c_{1}=\varepsilon_{1}\left|h_{S R_{i}}\right|^{2} \sqrt{P_{s}}, c_{2}=\varepsilon_{2}\left|h_{R D}\right|^{2} \sqrt{P_{R}}, \varepsilon_{1}$ and $\varepsilon_{2}$ are the energy consumed at the source and destination respectively. Now we consider $c_{1}=c_{2}=c$. Then the PDF can be expressed as [15].

$$
f_{\gamma_{i}^{u p}}(\gamma)=\frac{2 \gamma^{m-1} e^{\frac{-m \gamma}{c \bar{\gamma}_{c}}}}{\Gamma(m)}\left(1-\frac{1}{\Gamma(m)} \Gamma\left(m, \frac{m}{c \bar{\gamma}_{C}} \gamma\right)\right)
$$

We derive the MGF of $\gamma_{D}^{u p}$, using the PDF of $\gamma_{i}^{u p}$ in (21) as follows:

$$
M_{\gamma_{i}}(s)=\int_{0}^{\infty} e^{-\gamma s} f_{\gamma_{i}^{u p}}(\gamma) \cdot d \gamma
$$

We get the $M_{\gamma_{i}}(s)$ by solving the integral, the equation in (22) is attained, with ${ }_{2} F_{1}(:,:,:,:)$ symbolizing the Gauss hypergeometric function [16, eq (9.100)], of as follows:

$$
\begin{gathered}
M_{\gamma_{i}}(s)=\prod_{i=1}^{R}\left((m-1) !\left(\frac{c \bar{\gamma}_{c}}{m}-S\right)^{m}-\right. \\
\left.\frac{1}{\Gamma(m)} \frac{\left(m / c \bar{\gamma}_{C}\right)^{m} \Gamma(m)}{m\left(2 m / c \bar{\gamma}_{C}+s\right)^{2 m}}{ }_{2} F_{1}\left(1,2 m, m+1, \frac{m}{2 m+c \bar{\gamma}_{C} S}\right)\right)
\end{gathered}
$$

Let the average SER for M-PSK signals can be written as [14].

$$
P_{S E R}=\frac{1}{\pi} \int_{0}^{\frac{(M-1) \pi}{M}} M_{\gamma_{D}^{u p}}(s)\left(\frac{g_{M-P S K}}{\sin ^{2} \theta}\right) d \theta
$$

where $g_{M-P S K}=\sin ^{2}(\pi / M)$

For the upper SER expression, the upper bound value of the SER is obtained by using the maximum value of the integral:

$$
P_{S E R} \leq\left(1-\frac{1}{M}\right) M_{\gamma_{D}^{u p}}(s)\left(g_{M-P S K}\right)
$$

By combining MRC signals at the destination, the AF mode to obtain the maximum information of the end transmission rate.

$$
R_{m r c}=0.5 \log \left(1+\gamma_{D}\right)
$$

Where the $\gamma_{S R_{i}}, \gamma_{S D}$ and $\gamma_{R_{i} D}$ respectively, a relay link $S \rightarrow R_{i}$ and $R_{i} \rightarrow D$. And direct link $S \rightarrow D$, Link instantaneous SNR, when the system transmission speed

$R>R_{m r c}$ When, No matter in what encoding transmission error appears that interruption. So the next AF agreement outage probability can be approximated as:

$$
\begin{aligned}
& P_{m r c}=P_{r}\left(R>R_{m r c}\right) \\
= & \int_{0}^{2^{2 R}-1}\left[\left[1-\left[1-P_{r}\left(\gamma_{S R_{i}} \geq \gamma\right)\right]\left[1-P_{r}\left(\gamma_{R_{i} D} \geq \gamma\right)\right]\right]\right. \\
& \left.\times\left[f_{S D}\left(\gamma_{S D}\right)\right]\right] d \gamma_{S D}
\end{aligned}
$$

\subsection{Performance of SC Combination \\ System}

In this section, we study Cooperative Networks of AF over Nakagami-m fading Channels in relaying with SC at the destination. The channel state information operates in halfduplex mode. Let direct link $S \rightarrow D$, and a relay link $S \rightarrow R_{i}$ and $R_{i} \rightarrow D$. SC the total end to end of the SNR combination $\gamma_{S C}$ is given by

$$
\gamma_{S C}=\max \left(\gamma_{i}, \gamma_{R_{i} D}\right)
$$

So the outage probability of AF system at this time can be expressed by as

$$
\begin{aligned}
& P_{S C}=P_{r}\left(R>R_{S C}\right) \\
= & P_{r}\left(0.5 \log _{2}\left(1+\max \left(\gamma_{i}, \gamma_{R_{i} D}\right)\right)<R\right)
\end{aligned}
$$

The CDF we know that $\gamma_{S D}$ can be expressed by using [16, eq (8.352.4)] as, we have 


$$
F \gamma_{S D}(\gamma)=1-e^{-\left(\frac{m}{\gamma_{S D}}\right) \gamma} \sum_{i=0}^{m-1}\left(\frac{m}{\gamma_{S D}} \gamma\right)^{i} / \mathrm{i} !
$$

And for the relay channel a relay link $S \rightarrow R_{i}$ and $R_{i} \rightarrow D$, the PDF by total probability is given by.

$$
\begin{gathered}
F \gamma_{i}(\gamma)=P_{r}\left(\gamma_{i}<\gamma\right) \\
=P_{r}\left(\gamma_{R D}<\gamma\right) \int_{\gamma}^{\infty}\left(\Gamma\left(m, \frac{m}{\bar{\gamma}_{S R_{i}}^{2}} \gamma\right)\left(\frac{\left(\gamma_{R D}+1\right) \gamma}{\left(\gamma_{R D}-1\right)}\right) f_{\gamma_{R D}}(\gamma)\right) \mathrm{d} \gamma_{R D}
\end{gathered}
$$

we got The outage probability of the system From multiplying (30)and(31).

$$
\begin{aligned}
P_{S C} & =P_{r}\left(R>R_{S C}\right) \\
& =F \gamma_{S D}(\gamma) \times F \gamma_{i}(\gamma)
\end{aligned}
$$

\section{NUMERICAL RESULTS}

In this section, we present some numerical results to reveal the impact of different to use the MRC scheme due to the significant difference in its performance compared with the $\mathrm{SC}$ scheme, as shown in the simulation results.

Fig.2 The figure shows that the MRC scheme for average SER versus SNR of the cooperative system with M-PSK modulation signal, using the equation (25) for different values R. And we use 4-PSK and 64-PSK modulation signal, we can see that increasing the number of relays decreases the average SER.

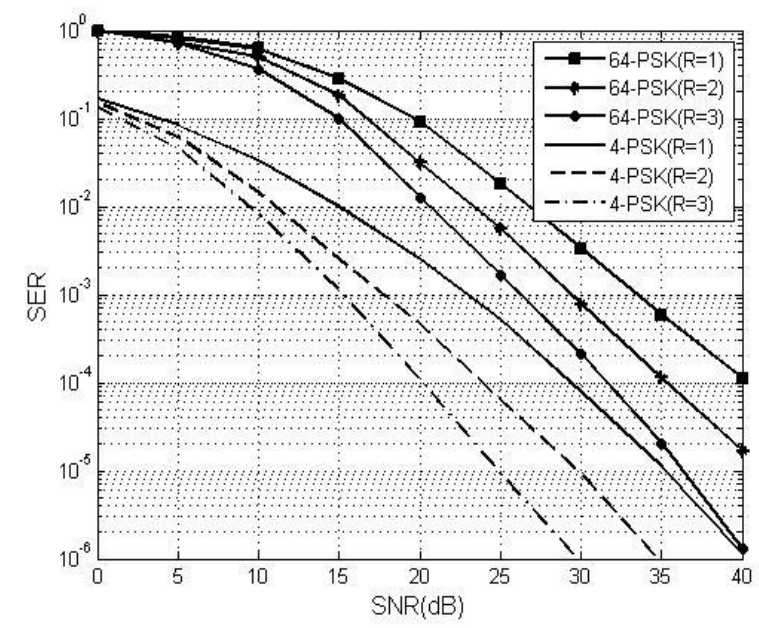

Fig.2 SER versus average SNR for M-PSK modulation signals over Nakagami-m with MRC

Fig.3 The figure shows that the SC scheme over Nakagami-m fading channels, and comparison of the SER performance for the indicating a direct impact on different modulation signals respectively with $R=4$ and $m=1$. It can be seen from the simulation that the performance of BPSK modulation is the best, and it has better performance under low SNR condition.

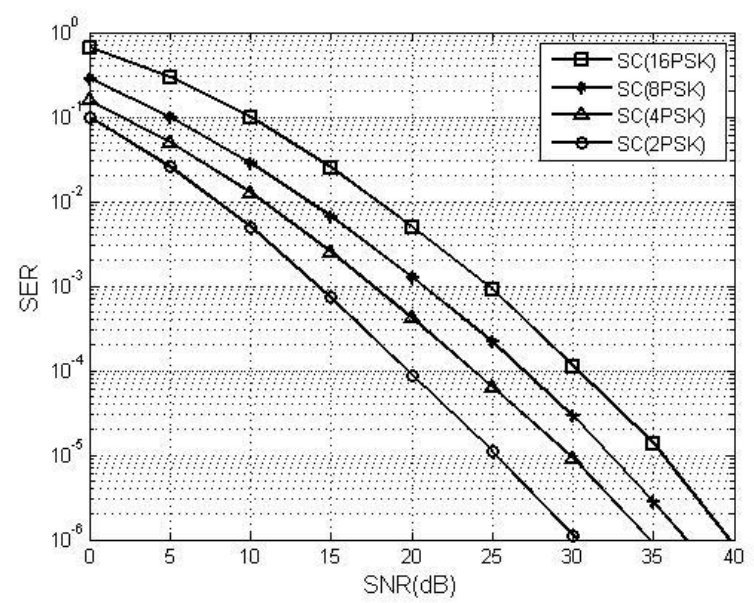

Fig. 3 Performance SER of AF protocol with SC over Nakagami-m when $(R=4, m=1)$

Fig.4 The figure shows that the SC scheme over Nakagami-m fading channels, with different number $(\mathrm{R}=1,2$ and 3$)$ relay system , and comparison of the SER performance for the two systems combined $\mathrm{R}=3$ under way when MRC and SC, This combined scheme has the advantages over the SC and because of the way that is easy to implement low complexity, so in high SNR conditions preclude the use of SC merger will also get better performance. Simulation parameters: QPSK modulation unit power signal, the relay number $\mathrm{R}=3$, Nakagami parameter $\mathrm{m}=1$.

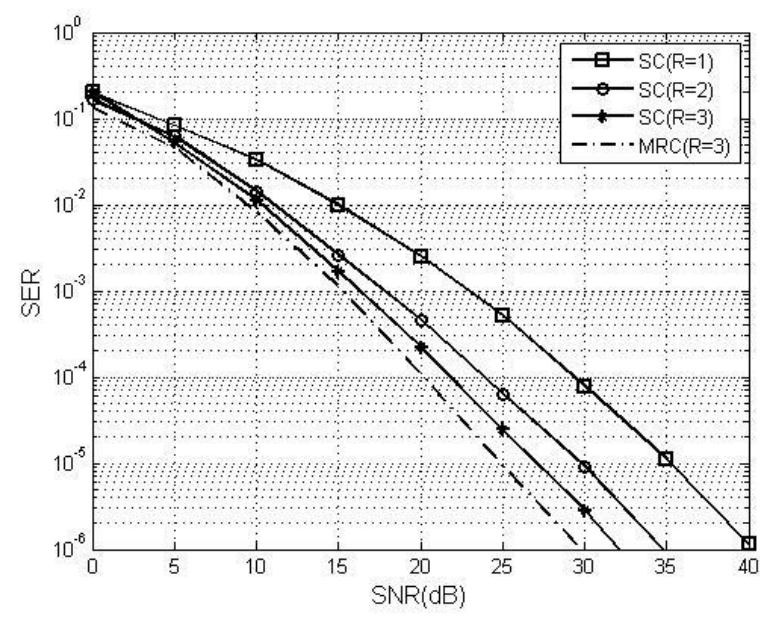

Fig. 4 Performance SER and compare MRC with SC over Nakagami-m when

\section{CONCLUSION}

In this paper, we have derived exact closed-form expressions for the PDF and the CDF for the channel state information operates in a half - duplex mode, we had also derived and analyzed indeed closed-form expressions for the SER and $\mathrm{P}_{\text {out }}$ over Nakagami-m fading channels with the MRC or SC combining schemes at the receiver in order to be used at the destination to achieve the desired diversity. New derived expressions for the outage probability and SER have been presented for both MRC and SC receivers at the destination. Furthermore, the simulation results proved that MRC scheme showing a better performance as compared with the SC scheme. The cooperative network that is considered to study the behavior of both MRC and SC schemes at the destination 
with M-ary phase-shift keying (M-PSK) and M-ary for different values of the ratio of the decision after a quadrature phase over Nakagami -m Fading Channels.

\section{ACKNOWLEDGMENTS}

This work was supported in part by the National High Technology Research and Development (863 project), No.2014AA01A701, 2015AA011303 and the National Natural Science Foundation of China under 61471408.

\section{REFERENCES}

[1] N. C. Beaulieu and Y. Chen, "An accurate approximation to the average error probability of cooperative diversity in Nakagami-m fading," IEEE Trans. Wireless Commun., vol. 9, no. 9, pp. 2707-2711, Sep. 2010.

[2] J. N. Laneman, D. N. C. Tse, and G. W. Wornell, "Cooperative diversity in wireless networks: Efficient protocols and outage behavior," IEEE Trans. Inf. Theory, vol. 50, no. 12, pp. 3062-3080, Dec. 2004.

[3] M. K. Simon and M.-S. Alouini, Digital Communication over Fading Channels, 2nd ed. New York: Wiley, 2005.

[4] Lei X, Beaulieu NC and Fan P. Precise MGF performance analysis of amplify-and-forward cooperative diversity in Nakagami-m fading. In Information Theory. CWIT. 11th Canadian Workshop on 2009 May 13 (pp. 13-16). IEEE.

[5] M. Nakagami. The m-distribution, a general formula of intensity distribution of rapid fading. in Statistical Methods in Radio Wave Propagation, W. G. Hoffman, ed. Pergamon Press, 1960; 3-36.

[6] O. Waqar, D. C. McLernon, and M. Ghogho, "Performance analysis of non-regenerative opportunistic relaying in Nakagami-m fading," in Proc. IEEE Personal, Indoor Mobile Radio Commun. Conf. (PIMRC), Sep. 2009, pp. 231-235.

[7] Chu, Shao-I. Performance of Amplify-and-Forward Cooperative Diversity Networks with Generalized Selection Combining over Nakagami-m Fading
Channels. IEEE Communications Letters, May.2012.Vol. 16, No.5: 634-637.

[8] Ali A. Mohammed, Li Yu, Manar Al-Kali and Desheng Wang. Analysis of Amplify-and-Forward Cooperative Networks with Nakagami-m fading Channels for MRC Diversity Combining. TELKOMNIKA Indonesian Journal of Electrical Engineering, 2015, 16(3):546-552.

[9] Khan, I., Rajatheva, N., Tanoli, S. A., \& Jan, S. (2014). Performance analysis of cooperative network over Nakagami and Rician fading channels. International Journal of Communication Systems, 27(11), 2703-2722.

[10] Z Chen, Z Chi, Y Liand B Vucetic. Error performance of maximal-ratio combining with transmits antenna selection in flat Nakagami-m fading channels. IEEE Trans. Wireless Commun. Jan. 2009; 8 (1): 424-431.

[11] Z. Chen, J. Yuan, and B. Vucetic . Analysis of transmit antenna selection/maximal-ratio combining in Rayleigh fading channels. IEEE Trans .Vehicular Technology. July. 2005; 54(4): 1312-1321.

[12] Alam, A. S. S., and GoangSeog, C. Performance of MQAM, M-DPSK and M-PSK with MRC diversity in a Nakagami-m fading channel. Journal of Central South University. 2014; 21(4): 1347-1352.

[13] S. Ikki and M. H. Ahmed. Performance analysis of cooperative diversity wireless networks over nakagami $\mathrm{m}$ fading channel. IEEE Commun. Letters. April. 2007; 11(4): 334-336.

[14] A. Papoulis, Probability, Random Variables, and Stochastic Process.McGraw-Hill, 1991.

[15] Ali A. Mohammed, Li Yu, and Manar Al-Kali. Performance Analysis of Amplify-and-Forward Cooperative Networks in Non-Identically Distributed Nakagami-m Channels with Best Relaying Selection. Journal of Communications. 2015; 10(6): 396-402.

[16] I. S. Gradshteyn and I. M. Ryzhik, Table of Integrals, Series and Products, 6th ed. New York, NY, USA: Academic Press, 2000. 